\title{
Aplicación de un ciclo de mejora en Historia de la Filosofía. Una propuesta de innovación docente en el aprendizaje de la filosofia antigua: metodología, contenidos y actividades
}

Application of a Cycle of Improvement in History of Philosophy. A Proposal for Teaching Innovation in the Learning of Ancient Philosophy: Methodology, Contents and Activities 
Jesús FERNÁNDEZ MUÑoz

ORCID: https://orcid.org/0000-0002-1706-5009

Universidad de Sevilla

Departamento de Estética e

Historia de la Filosofía

jesusfdez@us.es

Fecha de recepción: 20-11-2019

Fecha de aceptación: 22-11-2019

DOl: http://dx.doi.org/10.12795/9788447221912.087

Pp.: 1961-1981 
En este trabajo se analiza el desarrollo de un Ciclo de Mejora en el Aula (CIMA) que se ha llevado a cabo en la asignatura de Historia de la Filosofía Antigua del Grado en Filosofía de la Universidad de Sevilla. En el desarrollo del CIMA se han formulado los contenidos a partir de problemas y preguntas estructurantes, la metodología se ha modificado y se han realizado diversas actividades de contraste y prácticas con los denominados presocráticos. Todo esto partiendo de un modelo didáctico centrado en los estudiantes como eje central del aprendizaje, es decir, considerando a los alumnos como sujetos pensantes. Para desarrollar este ciclo se realizó un cuestionario inicial y otro final que muestran los resultados del aprendizaje y que además continúan con otra actividad complementaria al ciclo.

Palabras-clave: Historia de la Filosofia Antigua, Grado en Filosofia, docencia universitaria, experimentación docente universitaria, Ciclo de Mejora en el Aula.

\section{Abstract}

This paper analyzes the development of a Classroom Improvement Cycle (CIMA) in the subject of History of the Ancient Philosophy of the Degree in Philosophy of the University of Seville. In the development of the CIMA, the contents have been adapted based on problems and structuring questions, the methodology has been modified and various contrast and practical activities have been with themes with the so-called presocratics. All this based on a didactic model focused on students as the central axis of learning, that is, considering students as thinking subjects. To develop this cycle, an initial and final questionnaire was carried out to contrast the results of the CIMA that show the learning results and also continue with another activity complementary to the cycle.

Key-words: History of Ancient Philosophy, Degree in Philosophy, university teaching, university teaching experimentation, Classroom Improvement Cycle. 


\section{Introducción: descripción del contexto.}

El Ciclo de Mejora en el Aula (CIMA) se ha aplicado en la asignatura "Historia de la Filosofía Antigua" (turno de mañana) del primer curso del Grado en Filosofía de la Universidad de Sevilla del curso 2019-2020. Esta asignatura es de formación básica del Grado en Filosofía y se imparte en el primer cuatrimestre. Es la primera asignatura de Historia de la Filosofía que los alumnos cursan en dicho Grado Universitario y, por tanto, la labor introductoria a la materia de Historia de la Filosofía es doblemente importante.

El CIMA ha tenido una duración total de 8 horas tal y como se expone en el siguiente apartado. A lo que le han precedido 4 horas de un ciclo de mejora metodológico y le han seguido 6 horas de actividades complementarias sobre los mismos temas realizadas por los alumnos (exposiciones en grupo). Los temas en los que se ha aplicado el CIMA estaban relacionados con los presocráticos siguientes: Heráclito de Éfeso, Parménides de Elea, Empédocles de Acragas y Anaxágoras de Clazomene. Y en las actividades complementarias se han añadido la escuela de Mileto (Tales, Anaximandro y Anaxímenes), Pitágoras de Samos (y los pitagóricos) y Leucipo y Demócrito (los atomistas).

En este trabajo se analizará la metodología general llevada a cabo (Figura 2), el mapa de contenidos completo (Figura 1) y se ejemplificará todo ello con el tema dedicado a Anaxágoras por una cuestión de limitación del espacio disponible. Por lo tanto, del tema de Anaxágoras se expondrá tanto la secuencia de actividades como la escalera de aprendizaje resultante de la comparación entre el cuestionario inicial y el final, así como su mapa de contenidos específico. 


\section{Diseño del Ciclo de Mejora en el Aula. Los contenidos, la metodología y las actividades.}

El mapa de contenidos y problemas que se presentan en este CIMA se refiere a los 3 temas que se impartirán en las cuatro sesiones de dos horas cada una (total 8h) en los que se incluye este ciclo de mejora. En cada sesión se tenía previsto impartir las ideas principales (y los problemas que estos plantean) y cómo le da soluciones cada filósofo presocrático. En el primer caso, el tema 5 se impartió en dos sesiones ( 4 horas) y en el segundo y tercero en una sesión cada tema $(2+2$ horas). Los temas van del 5 al 7 y según el proyecto docente de la asignatura y son los siguientes:

Tema 5. Discusión en torno al problema del cambio: de Heráclito a Parménides y los Eléatas.

Tema 6. Empédocles: las cuatro raíces y los ciclos cósmicos.

Tema 7. Anaxágoras: radicalización del pluralismo, mecanicismo y teleología

Se ha preparado un mapa de contenidos general donde se vinculan los primeros filósofos presocráticos con el problema fundamental del origen de la naturaleza y sus relaciones con los temas del CIMA. Este problema está incardinado en el "contenido estructurante" que se fundamenta en tres cuestiones centrales:

1) la unidad de la diversidad que es la novedad de estos pensadores, es decir, que plantean un sentido a la diversidad de la realidad.

2) el arjé en cada uno de los casos, es decir, el elemento originario del que se forma toda la realidad.

3) los principios de movimiento y del cambio a partir de los que se forma el mundo y la realidad circundante. 
De la misma manera, los problemas se vinculan con dicho contenido estructurante. Por tanto, el mapa conceptual no solo se ha centrado en los tres temas del CIMA, sino que se han indicado las relaciones (más sintéticamente) con otros presocráticos, así como sus influencias. Esto se ha planteado así para contextualizar dicho mapa de contenidos de una forma coherente en su contexto histórico-filosófico. 


\section{Jesús FERnÁNDEZ MUÑoZ}

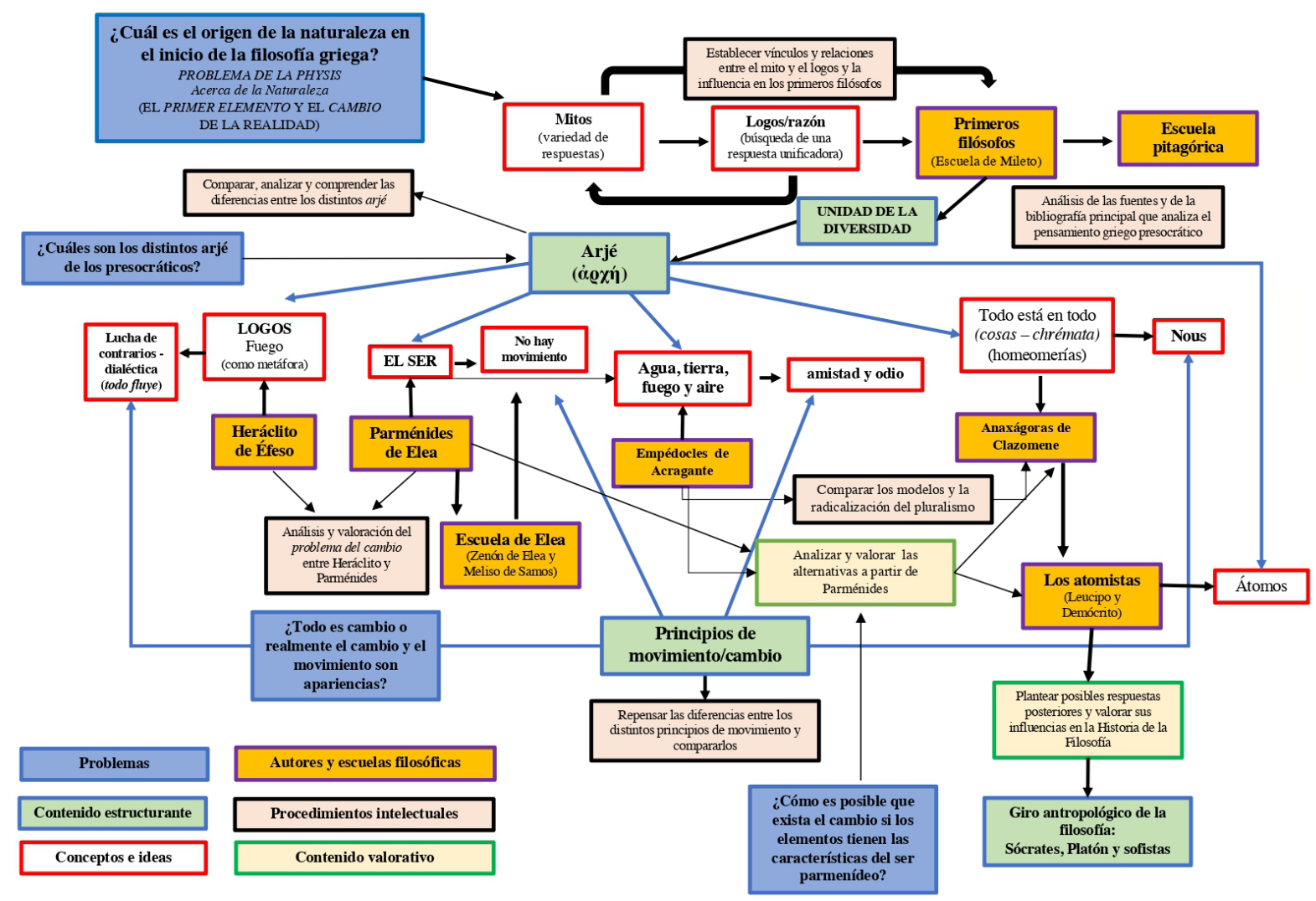

Figura .1Mapa de contenidos

Jornadas de Formación e Innovación Docente del Profesorado | № 2 (2019) Esta obra se distribuye con la licencia Creative Commons Reconocimiento-NoComercial-SinObraDerivada Internacional (CC BY-NC-ND 4.0.)

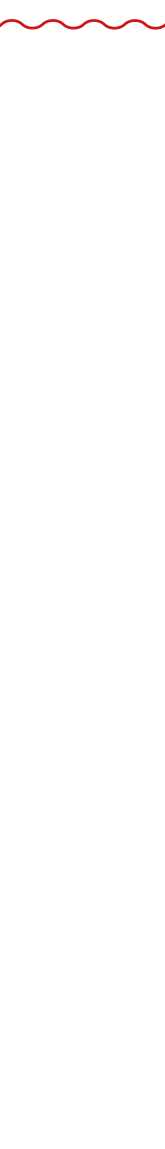


Además de este mapa general de contenidos (Figura 1) se han confeccionado cuatro mapas de contenidos particulares centrados en cada filósofo presocrático, sin embargo, en este trabajo solo se expone el que tiene que ver con Anaxágoras (Figura 3).

El modelo metodológico posible que se diseñó y se realizó en el primer CIMA (metodológico) de 4 horas ha servido para perfilar y mejorar el modelo metodológico del presente CIMA que es el siguiente:

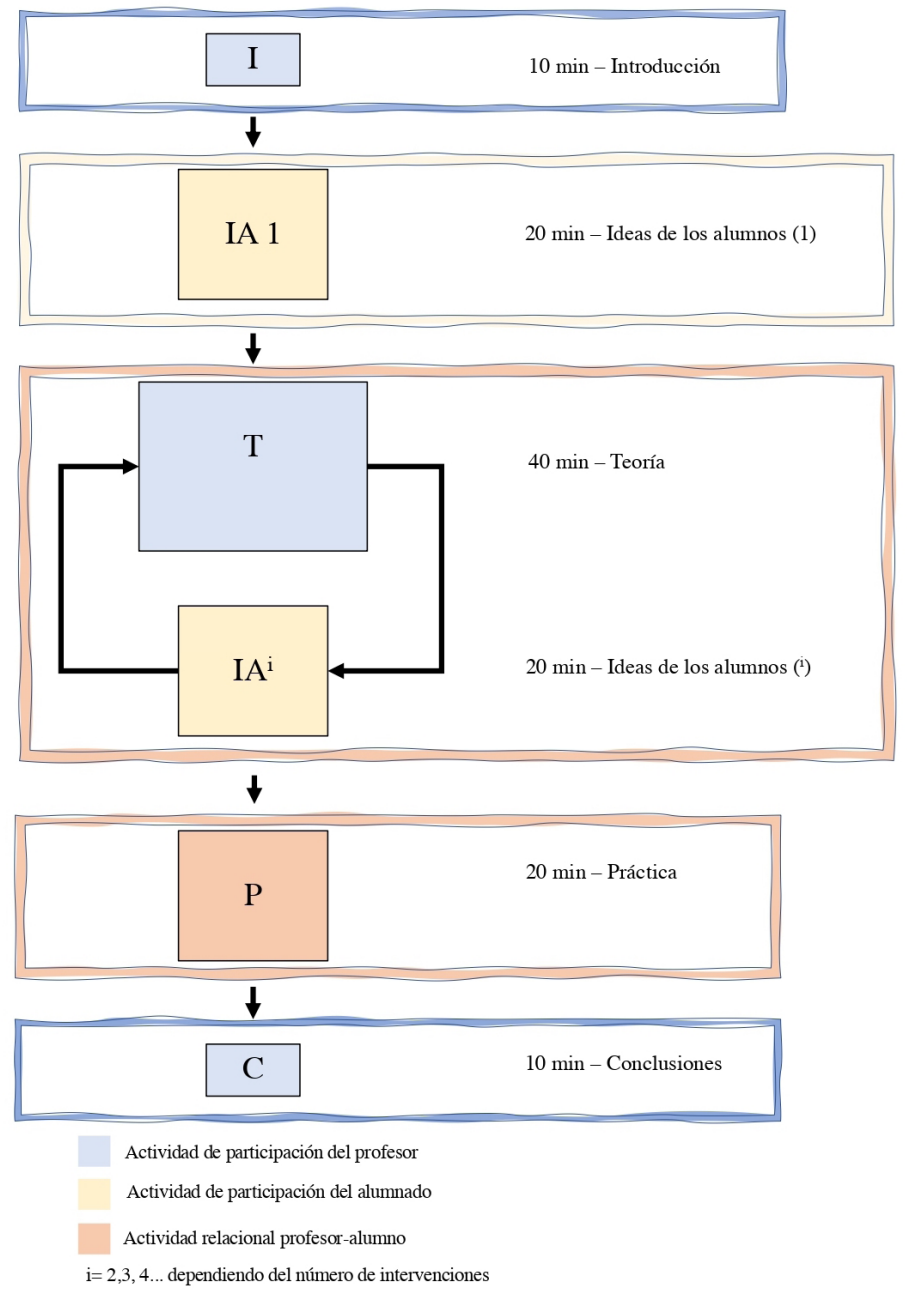

Figura 2. Modelo metodológico

Jornadas de Formación e Innovación Docente del Profesorado | № 2 (2019) Esta obra se distribuye con la licencia Creative Commons Reconocimiento-NoComercial-SinObraDerivada 4.0 Internacional (CC BY-NC-ND 4.0.) 
La justificación y explicación del modelo es la siguiente:

1) Se fundamenta en la importancia que tiene ir de lo general (ideas previas que los alumnos conocen y dominan) a otros aspectos más concretos (ideas especializadas y conceptos filosóficos que permitirán mayores abstracciones y relaciones de ideas más complejas) de manera escalonada y progresiva.

2) Para comprender las cuestiones o temas concretos y particulares es necesario que el alumno obtenga previamente una panorámica general y amplia (introductoria y contextual). De ahí la importancia de la Introducción (I) y del planteamiento de problemas y sub-preguntas relacionadas desde el inicio. Si se problematizan los temas desde el principio a partir de preguntas el alumno podrá seguir el hilo conductor de todo el contenido y tendrá algunas herramientas necesarias para ser capaz de ir construyendo los vínculos temáticos.

3) Las Ideas de los Alumnos (IA1) son parte fundamental para que los estudiantes muestren, a partir de un problema y sus diversas preguntas particulares en la I, lo que saben y las relaciones temáticas que realizan, así como las respuestas a los problemas y preguntas y el debate que se genera entre los propios alumnos.

4) La Teoría $(T)$ es una parte central en el modelo metodológico pero vinculada con las demás de manera completa. De hecho, tiene una relación total con las IA debido a que se desarrolla a partir de distintas preguntas, problemas y relaciones con las ideas de los alumnos (que son indeterminadas) así como con los problemas que le va planteando dicha teoría. Una teoría que se va modificando y replanteando durante esta fase, es decir, hay una T y unas IA en constante 
relación y cambio, de manera que la T va adaptándose al nivel del alumnado.

5) La parte Práctica (P) consiste en aplicar los contenidos teóricos aprendidos a través de distintas actividades y con la ayuda, contraste y retroalimentación del profesor.

6) Conclusiones (C) breves en la que se sintetizan los aspectos más importantes de la sesión.

En otras palabras y de manera sintética, el sentido de este esquema metodológico es el progreso gradual, que parte de las IA y los conocimientos de los alumnos, para ir guiando, construyendo y reconstruyendo desde la base general de los estudiantes. Un proceso diseñado para que los alumnos sean (realmente) los protagonistas de su aprendizaje y, por tanto, la parte activa central en el aula (y fuera de ella con las tareas entre sesiones).

Para organizar las clases y ver el nivel del que los estudiantes partían se diseñó un cuestionario inicial (Tabla 1) del que posteriormente se analizarían de manera sistemática las respuestas de los alumnos y se contrastó con las respuestas del mismo cuestionario final después de que terminase el CIMA para, de esta manera, establecer la comparación tal y como aparece en la figura 3 que corresponde a la escalera de aprendizaje.

El cuestionario (Tabla 1) se confeccionó de una manera atractiva y distendida para que los estudiantes no tuviesen la sensación de que se encontraban en un examen o similar. Lo que se pretendía era poner en un contexto relajado y que pudiesen responder con tranquilidad desde sus ideas iniciales y relaciones lógicas que tuviesen en cuanto a las preguntas que se planteaban. 
Con una máquina del tiempo mágica te trasladas a Mileto en el año 540 a.C. (comprendiendo perfectamente la lengua que allí se habla) y allí escuchas hablar de las teorías fisicas y filosóficas de la escuela de Mileto: sobre Tales, Anaximandro y Anaxímenes. Te cuentan, aunque no de primera mano, qué cosas pensaba cada uno de ellos e incluso has conseguido ver a Anaximenes de lejos, algo que te emociona sobremanera porque quieres decirle que se equivoca y que su maestro Anaximandro con su ápeiron tenía una teoría más completa que la suya. En tu estancia en el s. VI a. C. conoces, al menos de oídas, las teorías de los milesios que relacionan la diversidad de la realidad (de la naturaleza) en una unidad (arjé), pero que no se paran a explicar el problema del cambio demasiado bien. Por otro lado, te has dado cuenta de la íntima relación que existe entre el mito y el logos. Y, pese a lo que te contaron en bachillerato, constatas que las teorías de estos primeros filósofos tienen mucha relación con cuestiones míticas (el mito no se ha desligado del logos). De manera imprevista, y por un fallo de tu máquina del tiempo, te transportas a Atenas y vas hacia adelante algunos años (409 a.C.). De repente te encuentras con el joven Platón (iqué casualidad!), que tiene tu misma edad, y que cuando te ve aparecer de la nada se sorprende y piensa que eres un ser divino (daimón). Empiezas a hablar con él y le cuentas brevemente tu historia: le añades que acabas de estar en Mileto de hace más de cien años y que conoces bien esas teorías (?). Entonces Platón comienza a hacerte algunas preguntas:

1. Dime, por favor, qué otros elementos, además del agua (Tales), ápeiron (Anaximandro) y aire (Anaximenes) has escuchado que pueden ser los constitutivos de la realidad. ¿Cómo podrían moverse esos elementos (causa del movimiento)? Si no has escuchado hablar de otros elementos ¿cuáles se te ocurren a ti? ¿qué elementos podrían constituir realmente la realidad?

2. Me ha dicho mi maestro Sócrates que había dos sabios que hablaban del problema del cambio (Heráclito y Parménides), uno pensaba que el movimiento no existe (es apariencia) y que era una ilusión y el otro que todo estaba en constante cambio. ¿Qué piensas al respecto? ¿todo es cambio o realmente el cambio y el movimiento son apariencias?

3. He escuchado hablar de un tal Empédocles que afirmaba que la realidad se componía de cuatro elementos y le otorgaba las características del ser parmenídeo ¿qué sabes sobre eso? Porque si tenían las características del ser de Parménides significaría que no hay cambio, sin embargo, él habla de cambio ¿cómo puede ser eso posible? ¿cómo lo entiendes tú? 
4. Todo está en todo y existe la divisibilidad infinita de la materia. Eso decía un filósofo llamado Anaxágoras e indicaba que todo se movía gracias al Nous (Intelecto). Y para rizar el rizo, insistía, como Parménides, en que unas cosas no cambian para convertirse en otras distintas, es decir, que todas las cosas eran eternas e inengendradas (una cosa $A$ no puede convertirse en cosa $B$ ) y, sin embargo, por lo visto, existe el cambio y él constata que hay cosas diferentes en la realidad ¿qué puede significar realmente todo esto?

Una vez realizado el análisis de las respuestas cuestionario inicial -a un total de 37 estudiantes- se preparó la secuencia de actividades de cada sesión. Todo ello a partir de planteamientos adaptados a sus conocimientos iniciales según las respuestas que habían dado en el cuestionario.

En este trabajo, como se ha indicado, se exponen en detalle la secuencia de actividades de una sesión en particular (Tabla 2), la de Anaxágoras, así como el mapa de contenidos (Figura 3) del mismo filósofo presocrático de Clazomene. Las otras secuencias de actividades estaban diseñadas de manera idéntica pero adaptadas al tema en cuestión, por lo tanto, esta secuencia y mapa de contenidos puede servir de ejemplo.

Jornadas de Formación e Innovación Docente del Profesorado | № 2 (2019) Esta obra se distribuye con la licencia Creative Commons 


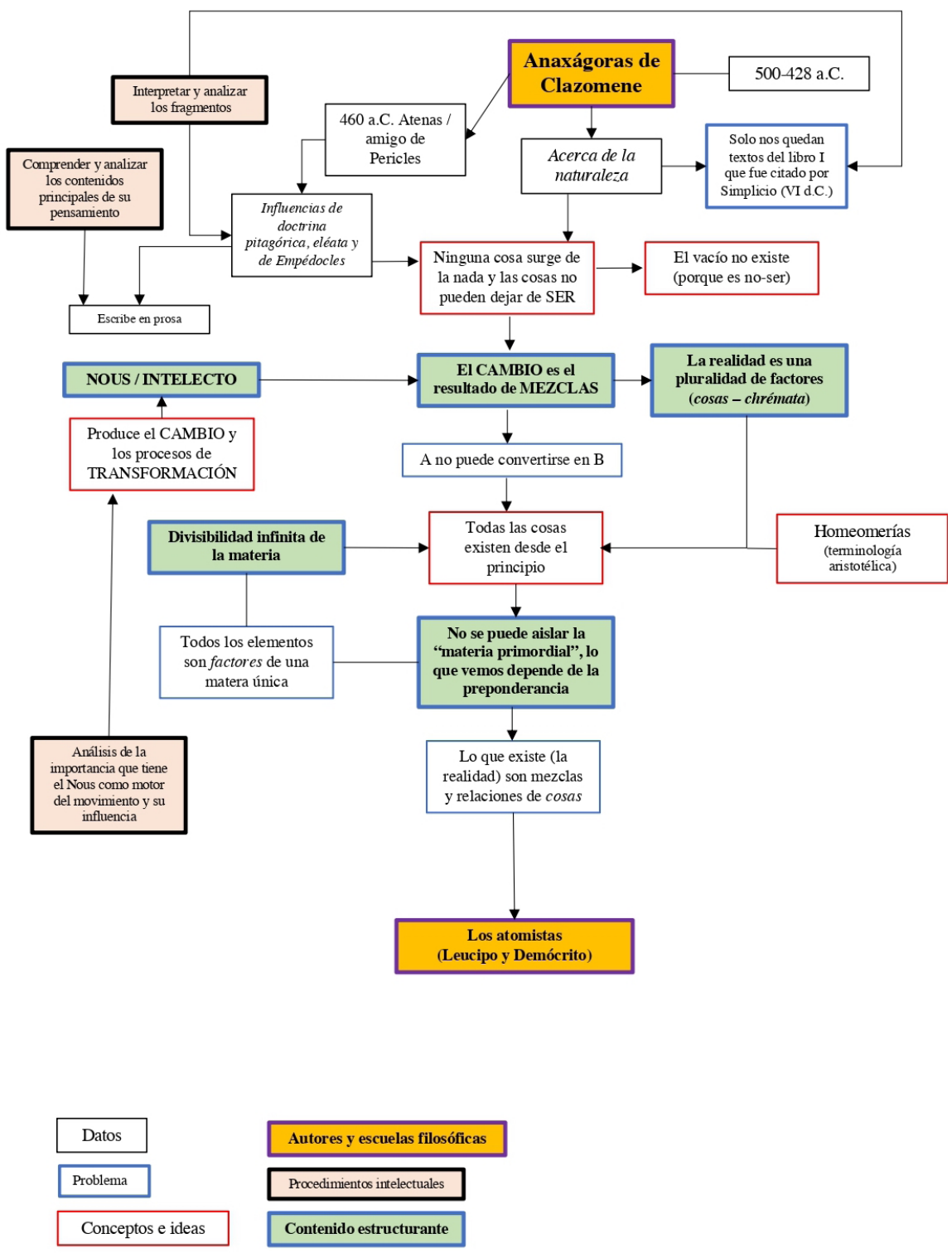

Figura 3. Mapa de contenidos de Anaxágoras

Jornadas de Formación e Innovación Docente del Profesorado | № 2 (2019)

Esta obra se distribuye con la licencia Creative Commons

Reconocimiento-NoComercial-SinObraDerivada

Internacional (CC BY-NC-ND 4.0.) 


\begin{tabular}{|c|c|c|}
\hline \multicolumn{3}{|c|}{ SESIÓN 4. ANAXÁGORAS DE CLAZOMENE (2 h) } \\
\hline Actividad & Descripción & Tiempo \\
\hline 1. Introducción (I) & $\begin{array}{l}\text { 1.1. Planteamiento de problema general: Todo está en } \\
\text { todo y existe la divisibilidad infinita de la materia. Eso } \\
\text { decía Anaxágoras e indicaba que todo se movía gracias } \\
\text { al Nous (Intelecto). Por otro lado, también insistía, } \\
\text { como Parménides, en que unas cosas no cambian para } \\
\text { convertirse en otras distintas, es decir, que todas las } \\
\text { cosas son eternas e inengendradas (una cosa A no puede } \\
\text { convertirse en cosa B) y, sin embargo, existe el cambio y } \\
\text { él constata que hay cosas diferentes en la realidad ¿qué } \\
\text { puede significar realmente todo esto? ¿qué significa que } \\
\text { todo está en todo? ¿cómo puede la materia dividirse } \\
\text { infinitamente? } \\
\text { 1.2. Sub-problema: ¿es necesario que exista un primer } \\
\text { motor del universo? } \\
\text { 1.3. Contextualización histórico-filosófica de Anaxágoras } \\
\text { (sin entrar en la filosofia ni en el pensamiento) } \\
\text { 1.4. Recomendación de la bibliografía básica y } \\
\text { complementaria y los apartados más relevantes del } \\
\text { pensamiento de Anaxágoras y relación con otros } \\
\text { presocráticos (coetáneos y posteriores: especialmente } \\
\text { con Empédocles y los atomistas). } \\
\text { 1.4. Preguntas particulares sobre Anaxágoras: ¿qué } \\
\text { sabéis sobre Anaxágoras? ¿qué es lo fundamental de su } \\
\text { pensamiento? ¿qué son las homeomerías y cuáles son sus } \\
\text { características? }\end{array}$ & $10 \mathrm{~min}$. \\
\hline $\begin{array}{l}\text { 2. Ideas de los } \\
\text { alumnos } 1 \text { (IA 1) }\end{array}$ & $\begin{array}{l}\text { 2.1. Los alumnos expondrán sus ideas acerca de Anaxágoras } \\
\text { a partir de lo que le han enseñado en bachillerato o lo } \\
\text { que hayan estudiado o leído por su cuenta, así como por } \\
\text { lo que han aprendido del vídeo introductorio al tema. } \\
\text { 2.2. Todas las ideas y conceptos que los alumnos planteen } \\
\text { se escribirán en la pizarra y los propios alumnos indicarán } \\
\text { las relaciones entre sí (si las hubiere). }\end{array}$ & 20 min. \\
\hline
\end{tabular}




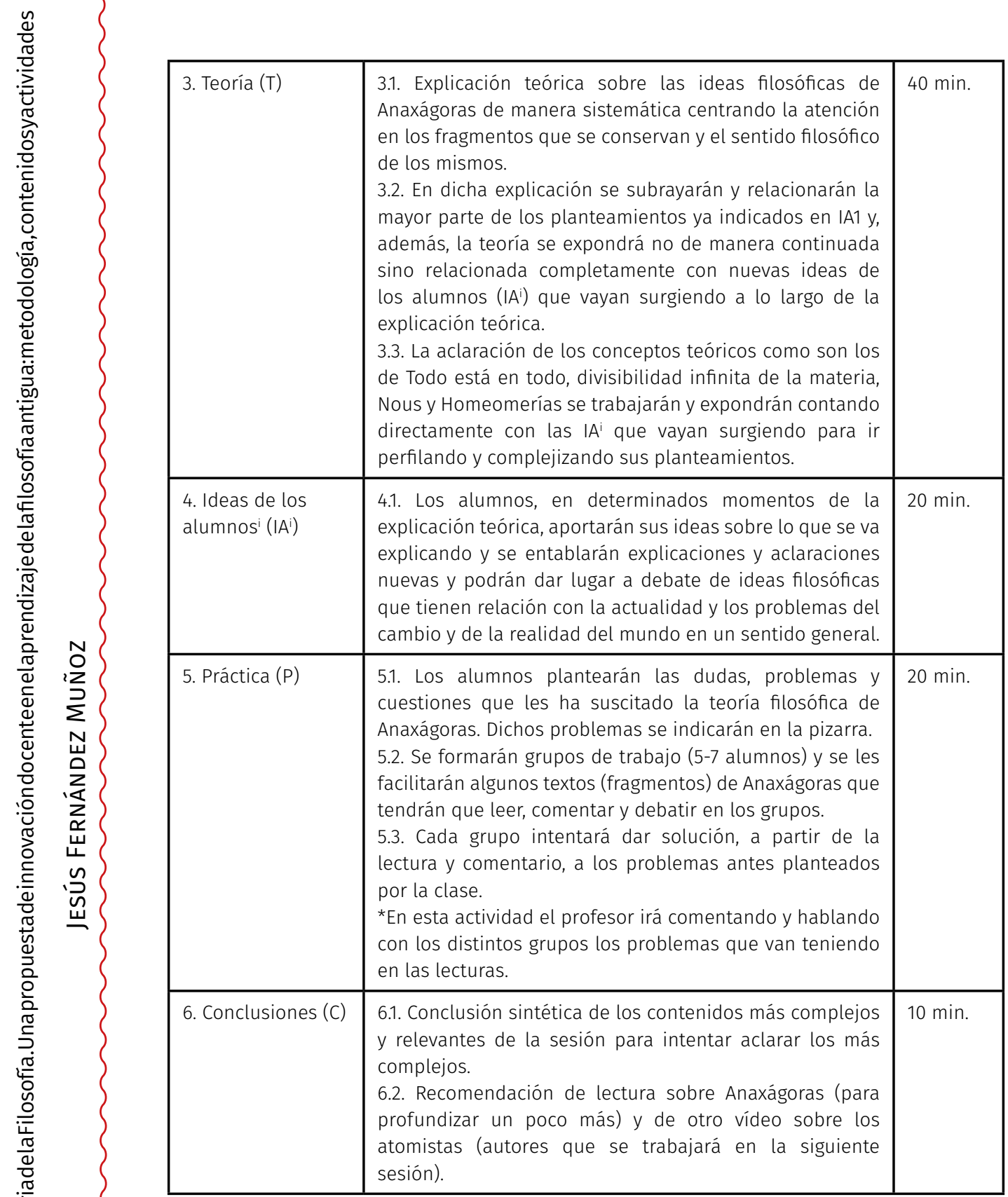

La secuencia de actividades se corresponde exactamente con el modelo metodológico planteado y tiene una relación interna continuada a lo largo de la sesión, es decir, 
no son momentos aislados, sino que se han planteado desde la relación total y para que no impliquen cortes o interrupciones entre las diferentes partes. Esto significa que los estudiantes lo percibirán como un continuum.

\section{Aplicación del Ciclo de Mejora en el Aula y evaluación del aprendizaje}

Todas las sesiones han seguido el mismo modelo metodológico. En todos los casos las sesiones se han ido adaptando a las necesidades particulares de los estudiantes en cada caso, dependiendo de cada autor (filósofo) tratado. Porque el eje en torno al que han girado todas las sesiones, como ya se indicó, han sido los alumnos (IA) y los problemas planteados en el mapa de contenidos, así como las preguntas guía.

De esta manera todas las sesiones han comenzado con una introducción de unos 10 minutos donde se planteaban los problemas principales que los alumnos tenían que resolver, todos ellos muy vinculados a varias preguntas. En todas las sesiones las preguntas podían ser respondidas con las ideas de los alumnos, pero además todos deberían haber visto un vídeo recomendado en la sesión anterior para poder entender y captar más detalles en la sesión presente. Tras la introducción y planteamiento de los problemas se daba paso directamente a las Ideas de los Alumnos 1 (IA1), es decir, todas las ideas que tiene el grupo sobre los problemas a los que se han aludido, así como del autor en particular sobre el que girará la sesión. Esta parte de las IA1 ha sido en todos los casos muy participativa y ha dado lugar a multitud de ideas que se iban relacionando e interconectando entre ellos, como profesor me limitaba a escribir las ideas en la pizarra y realizar los vínculos que ellos iban comentado. En todos los casos el ambiente fue muy cómodo y la atención era total. 
A partir de este momento, y teniendo en cuenta todas esas ideas, se pasaba a la parte de la Teoría (T) que comenzaba directamente relacionando las IA1 con la T, es decir, de manera "improvisada" comenzaba a resolver los dilemas, planteamientos y dudas que tenían los alumnos desde el punto de vista teórico, pero relacionándolo con todo lo que habían dicho. Por lo tanto, aunque a partir de aqui se considera T, los alumnos en todas las sesiones pedian aclaraciones, planteaban nuevas ideas y proponían nuevos problemas. Esto significa que la parte de $\mathrm{T}$ se ha ido construyendo y retroalimentando constantemente de las IA (en un número indeterminado en cada sesión). Evidentemente, desde mi perspectiva le iba dando una sistematicidad a la explicación a la hora de desarrollar la T, pero desde la perspectiva del alumno no tenían esa impresión y, por tanto, se sentían parte del desarrollo y no dejaban de participar y la atención era muy alta. Esto significa que la parte de T y de IA estaban estrecha e indisolublemente vinculadas.

Cuando se pasaba a la parte Práctica $(P)$, es decir, al trabajo con los textos (fragmentos) de los filósofos, los alumnos ya tenían una idea "mejorada" y en gran parte pensada por ellos sobre los problemas de los autores y sobre los autores mismos. Esto implicaba que la lectura de los fragmentos no generaba tantos problemas, sino que, realmente, aclaraba muchos aspectos de este. En esta parte de $P$ he seguido diversas metodologías: en unos casos hacían lecturas en grupos y yo pasaba por los grupos y comentaba, en otro caso la lectura iba en orden y cada alumno iba leyendo en alto para toda la clase y comentaba en general qué pensaba al respecto y en otro caso la lectura fue más guiada por mi parte y yo leía el fragmento (aunque ellos lo tenían también delante y lo leían a la vez) para que lo comentasen. Todas las versiones de $\mathrm{P}$ han ido bien y en todas me he sentido cómodo y sin problema para el desarrollo. Se podría decir que todas las sesiones han fluido muy bien, los alumnos se han implicado muchísimo y el interés ha sido muy alto en todas ellas. 
Finalmente, las sesiones concluían (C) haciendo una sintesis de los contenidos principales tratados y de los respectivos problemas, así como dando algunas recomendaciones para la siguiente sesión.

Así las cosas, todas las sesiones han sido muy cómodas y alegres para mi y se podría decir que la alta participación, el interés de los alumnos y su implicación ha sido clave para ello. Si bien es cierto que en gran medida todo se ha debido, en parte, a que desde el primer día he desarrollado una "metodología" de trabajo que dejaba claro a los alumnos que eran protagonistas (de manera implícita y explícita) y que el fomento de la participación se ha dado desde la primera sesión del curso, lo que ha generado una confianza importante $y$, por ello, todo ha funcionado muy bien.

En general la implementación de este CIMA ha superado mis expectativas en todos los sentidos y el resultado ha sido muy bueno, me he sentido muy bien en esta dinámica de clases y muy motivado para realizar otras actividades que han implicado diversas exposiciones de los alumnos, también con muy buena respuesta y predisposición por parte de ellos. De hecho, para la evaluación de los estudiantes se ha continuado con esa serie de exposiciones que los alumnos diseñaban en grupos y que tenían que exponer al resto en un máximo de una hora. El resultado ha sido igualmente satisfactorio $y$, como se ha indicado, la participación ha sido elevada. Esa actividad tendrá un valor en la evaluación y, además, en el examen, los alumnos que hayan realizado la exposición tendrán que desarrollar en la prueba escrita el tema del que han realizado su exposición (un dato que no se les ha facilitado a ellos para que de cara al examen preparen el resto de los temas pero que, no obstante, les facilitará la labor en el desarrollo de la prueba). 


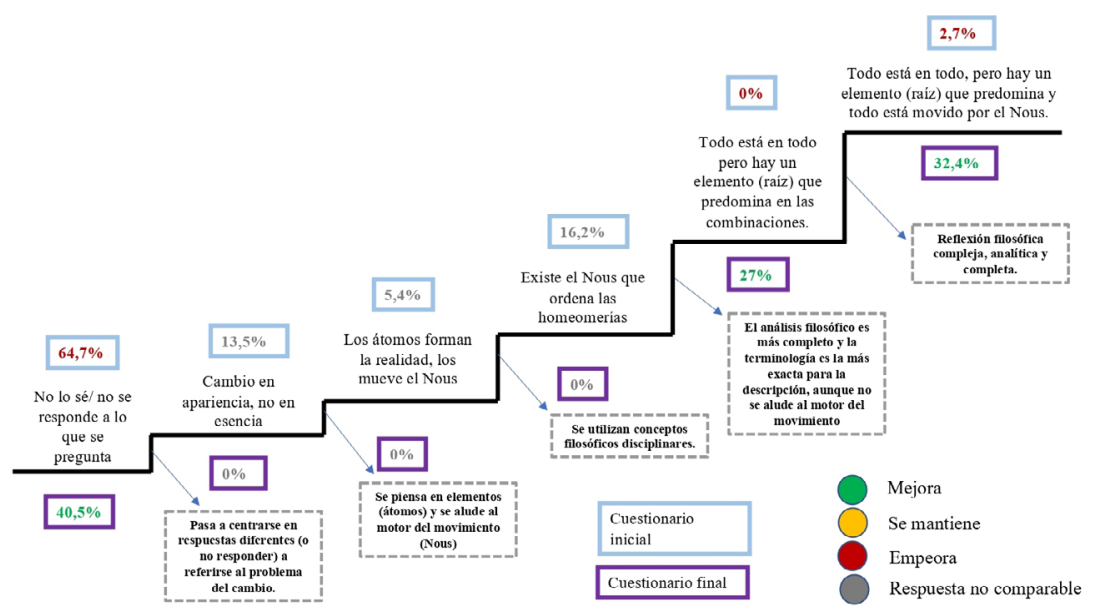

Figura 4. Escalera de aprendizaje del tema de Anaxágoras

En esta escalera de aprendizaje se puede comprobar que la diferencia entre el cuestionario inicial y el final es bastante significativa. Se puede apreciar que en el cuestionario final casi un $60 \%$ de los alumnos se sitúan en los peldaños más altos de la escalera, respondido de manera más completa y disciplinar a las preguntas. Es significativo el hecho de que todavía hay un $40 \%$ de alumnos que o no responde o responde a algo completamente distinto, sin embargo, ese porcentaje ha disminuido en casi un $25 \%$ respecto al inicial. Del mismo modo es interesante constatar que los peldaños intermedios en el cuestionario final están vacíos. Esto significa que la mayor parte de los alumnos se sitúan en una comprensión importante de los contenidos y una proporción menor continúa en la parte más baja.

\section{Evaluación del ciclo de mejora: principios docentes y didácticos}

Lo que mejor ha funcionado en este ciclo, además de los resultados del aprendizaje, ha sido el modelo metodológico posible planteado que fomentaba que los estudiantes fuesen el centro del proceso. Los alumnos han 
participado de manera general y han mostrado un interés muy elevado. De hecho, la asistencia a clase ha sido muy alta también. El hecho de que se haya podido llevar a cabo sin ningún problema es significativo y se debe a la que labor del alumnado era central. Eso quiere decir que la respuesta de los estudiantes ha sido la esperada en un sentido ideal y eso ha facilitado mucho el trabajo del CIMA. Entiendo que dicha participación e implicación de los alumnos se debe principalmente a su vocación por la filosofia, pero también al trabajo desde el primer día de clase por mi parte fomentando la participación, planteándoles problemas que les interesaban y estableciendo un vínculo de confianza e implicación mutua elevado. Todo esto los alumnos lo detectan muy rápidamente y en este caso han respondido muy bien. De hecho, en la actividad posterior al CIMA -que como se ha indicado consistía en unas exposiciones de los temas por parte de los estudiantes y en grupos- han tenido una participación casi total y con unos resultados muy buenos.

Lo que no ha funcionado bien han sido las tareas que se enviaban entre sesiones. Les pedía que entre cada sesión viesen un vídeo de YouTube del canal de la UNED sobre cada autor que se estudiaría en la siguiente sesión. Los vídeos tenían una duración de unos 20-25 minutos y la mayor parte de los alumnos no los veía. Habría sido importante que en vez de pedir que los "viesen" (sin más) haberles pedido una ficha de cada vídeo, que trajesen por escrito las ideas principales del vídeo y, además, darle un valor calificatorio (aunque fuese pequeño) a dichos comentarios de los vídeos.

Los aspectos principales que pueden mejorar en próximos ciclos son los siguientes:

1) Las actividades entre sesiones es lo primero que se puede mejorar de una manera sencilla, como he indicado: pidiendo a los alumnos que hagan un pequeño informe de cada vídeo para que posteriormente me lo entreguen y darle un valor calificatorio. 
2) Me parece importante que entre las sesiones también leyesen los fragmentos de los autores y los tuviesen trabajados para que indicasen las dificultades de estos.

3) Del mismo modo se podría mejorar que la participación en las sesiones fuese total (o casi total). Es decir, intentar que todos los alumnos que asisten a las sesiones participen. Y aunque la participación ha sido muy alta (un 60-70\% del total) me hubiese gustado que aquellos estudiantes más tímidos se animasen a exponer sus ideas.

Por último, de manera general mis principios docentes y didácticos se basan en utilizar una metodología que considera a los estudiantes como el centro de las sesiones $y$, por tanto, como los verdaderos protagonistas en el aula. También tiene una gran importancia la elaboración de mapas de contenidos con problemas centrales y preguntas estructurantes que sirven de motivación y son la manera de conectar desde el principio con los alumnos. De la misma manera, las secuencias de actividades tienen una relevancia importante porque se confeccionan a partir de un cuestionario inicial que sirve para saber en qué nivel se encuentran los estudiantes $y$, por tanto, teniendo el "diagnóstico" se puede aplicar el "tratamiento", es decir, a partir de conocer todo ello se adaptan las sesiones. Respecto a la evaluación se considera básico la denominada evaluación continua de los estudiantes que asisten regularmente a clase y se les da la posibilidad de realizar comentarios de texto, debates en clase, así como exposiciones de temas con la finalidad de que el proceso sea siempre gradual y de que todos los estudiantes progresen en los contenidos de la asignatura, sus conceptos, ideas, relaciones, etc. No se puede olvidar que la evaluación es un proceso continuo desde el inicio de la asignatura hasta el final e implica conocer el progreso de los alumnos y esto va mucho más allá de la simple calificación final. 


\section{Referencias bibliográficas:}

Alba, N. de y Porlán, R. (2017). "La metodología de enseñanza". En Porlán (Coord.) Enseñanza universitaria: cómo mejorarla (pp. 37-53). Sevilla: Ediciones Morata.

Bain, K. (2007). Lo que hacen los mejores profesores de universidad. Valencia: Universitat de València.

Finkel, D. (2008). Dar clase con la boca cerrada. Valencia: Universitat de València.

Giné Freixes, N. (2009), "Cómo mejorar la docencia universitaria: el punto de vista del estudiantado", Revista Complutense de Educación, 20(1), 117-134.

Rivero, A. y Porlán, R. (2017). "La evaluación en la enseñanza universitaria”. En Porlán (Coord.) Enseñanza universitaria: cómo mejorarla (pp. 73-91). Sevilla: Ediciones Morata. 